\title{
Bicavitary effusion secondary to liver lobe torsion in a dog
}

This article was published in the following Dove Press journal:

Veterinary Medicine: Research and Reports

20 April 2016

Number of times this article has been viewed

\author{
Zaheda Khan' \\ Kathryn Gates ${ }^{2}$ \\ Stephen A Simpson ${ }^{3}$ \\ 'Emergency and Critical Care, \\ Animal Specialty and Emergency \\ Center, Los Angeles, CA, ${ }^{2}$ Emergency \\ and Critical Care, Advanced Critical \\ Care, Emergency and Specialty \\ Services, Culver City, CA ${ }^{3}$ Emergency \\ and Critical Care, Southern California \\ Veterinary Specialty Hospital, Irvine, \\ CA, USA
}

\begin{abstract}
We described the diagnosis and successful treatment of pleural and peritoneal effusion secondary to liver lobe torsion in a dog. A 12-year-old female spayed Borzoi dog was referred for heart failure. Emergency room thoracic and abdominal ultrasound showed a large volume of pleural effusion with mild peritoneal effusion and an abdominal mass. Pleural fluid analysis classified the effusion as exudative. A complete ultrasound revealed mild peritoneal effusion and decreased blood flow to the right liver lobe. Other causes of bicavitary effusion were ruled out based on blood work, ultrasound, echocardiogram, and computed tomography. The patient was taken to surgery and diagnosed with caudate liver lobe torsion and had a liver lobectomy. At the 2-week postoperative recheck, the patient was doing well and there was complete resolution of the pleural effusion. Liver lobe torsion is a rare occurrence in dogs and can be difficult to diagnose. Clinical signs are nonspecific for liver lobe torsion and patients may present in respiratory distress with significant pleural fluid accumulation. When assessing patients with pleural and peritoneal effusion, liver lobe torsion should be considered as a differential diagnosis.
\end{abstract}

Keywords: pleural effusion, peritoneal effusion, hepatic torsion

\section{Case report}

A 12-year-old female spayed Borzoi dog weighing $25.5 \mathrm{~kg}$ was referred for heart failure. The dog had a history of weakness, anorexia, and diarrhea for 4 days. On initial presentation to the referring veterinarian, the patient was $5 \%$ dehydrated and had an irregularly irregular heart rhythm. A complete blood count (CBC) was normal and chemistry revealed elevated alanine aminotransferase (ALT) of $828 \mathrm{U} / \mathrm{L}$ (reference interval 10-100 U/L) and mild hyperbilirubinemia of $8.6 \mu \mathrm{mol} / \mathrm{L}$ (reference interval $1.7-10.3 \mu \mathrm{mol} / \mathrm{L})$.

At presentation to the referral hospital, the dog was mildly febrile $\left(39.2^{\circ} \mathrm{C}\right)$. Physical examination abnormalities included increased respiratory effort with increased bronchovesicular sounds bilaterally, decreased lung sounds ventrally, organomegaly, sinus tachycardia with pulse deficits, and a grade II/VI left systolic murmur. An initial indirect systolic blood pressure measured using a Doppler flow detector (Doppler Flow Detector Model 811-B; Parks Medical Electronics Inc., Aloha, OR, USA) was $110 \mathrm{mmHg}$ and pulse oximetry (SurgiVet ${ }^{\circledR}$ Parameter Advisor ${ }^{\circledR}$ Vital Signs Monitor; SurgiVet-Smiths Medical, Dublin, OH, USA) was 94\%. The patient was placed on continuous electrocardiogram (SurgiVet ${ }^{\circledR} 3$ Parameter Advisor ${ }^{\circledR}$ Vital Signs Monitor; SurgiVet-Smiths Medical) monitoring which showed persistent atrial
PO Box 48333, Los Angeles, CA 90048, USA

Email Zaheda.Khan@gmail.com
Veterinary Medicine: Research and Reports 2016:7 53-58

(c) (1) (9) 2016 Khan et al. This work is published by Dove Medical Press Limited, and licensed under Creative Commons Attribution - Non Commercial (unported, v3.0)

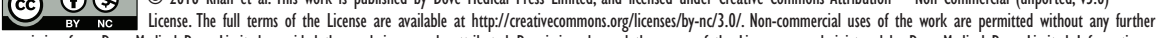
permission from Dove Medical Press Limited, provided the work is properly attributed. Permisions beyond the scope of the License are administered by Dove Medical Press Limited. Information on how to request permisision may be found at: http://www.dovepress.com/permissions.php

53
Dovepress

http://dx.doi.org/10.2147/VMRR.S83608

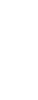


premature complexes (APCs) and ventricular premature complexes (VPCs) but no sustained tachyarrhythmia.

A repeat CBC (IDEXX Procyte $\mathrm{Dx}^{\circledR}$ Hematology Analyzer; IDEXX Laboratories Inc., Westbrook, ME, USA) was normal and chemistry (IDEXX Catalyst Dx ${ }^{\circledR}$ Chemistry Analyzer; IDEXX Laboratories Inc.) revealed severe elevation of ALT $>1,000 \mathrm{U} / \mathrm{L}$ (reference interval 10-100 U/L) and mild hyperbilirubinemia of $20.5 \mu \mathrm{mol} / \mathrm{L}$ (reference interval $0.0-15.4 \mu \mathrm{mol} / \mathrm{L}$ ). A coagulation profile revealed normal prothrombin time (IDEXX Coag Dx ${ }^{\mathrm{TM}}$ Analyzer; IDEXX Laboratories Inc.) and mild prolongation of activated partial thromboplastin time (IDEXX Coag Dx ${ }^{\mathrm{TM}}$ Analyzer; IDEXX Laboratories Inc.) of 120 seconds (reference interval 72.0-102 seconds).

Thoracic and abdominal focused assessment with sonography for trauma identified a large volume of pleural effusion bilaterally, mild peritoneal effusion, and a heterogeneous mass effect in the area of the right lateral liver lobe. Left-sided thoracocentesis yielded $700 \mathrm{~mL}$ of serosanguineous fluid with a packed cell volume (PCV) of $13 \%$ and total solids of $30 \mathrm{~g} / \mathrm{L}$. The patient was breathing comfortably after thoracocentesis and a right-sided thoracocentesis was not performed. Treatment included Clavamox (Zoetis, Florham Park, NJ, USA) $13.75 \mathrm{mg} / \mathrm{kg}$ per oral (PO) every 12 hours (q12h) empirically, and intravenous (IV) Lactated Ringer's solution (LRS) (Abbot Laboratories, Chicago, IL, USA) at $2.5 \mathrm{~mL} / \mathrm{kg} / \mathrm{hr}$, while awaiting fluid analysis and cytology. Fluid analysis reported an exudative fluid with a total protein of $35 \mathrm{~g} / \mathrm{L}$ and $14 \times 10^{9}$ cells/L. Cytology of the fluid revealed scattered nondegenerate neutrophils, vacuolated macrophages, and rare mesothelial cells. No intracellular or extracellular microorganisms were noted, and enrofloxacin (Bayer HealthCare LLC, Shawnee Mission, KS, USA) $5 \mathrm{mg} / \mathrm{kg}$ IV every 24 hours (q24h) was added.

The following day, the patient appeared to have increased respiratory effort and an additional $1,300 \mathrm{~mL}$ of serosanguineous fluid was removed via left-sided thoracocentesis. Recheck of ALT, total bilirubin, albumin, PCV, and total solids revealed an improving ALT of $509 \mathrm{U} / \mathrm{L}$ (reference interval 10-100 U/L), normal total bilirubin of $29.1 \mu \mathrm{mol} / \mathrm{L}$ (reference interval 0.0-8.6 $\mu \mathrm{mol} / \mathrm{L}$ ), decreasing albumin of $19 \mathrm{~g} / \mathrm{L}$ (reference interval 22-39 g/L), and total solids of $48 \mathrm{~g} / \mathrm{L}$, with a normal PCV of $39 \%$. A complete abdominal ultrasound revealed less (unable to sample) peritoneal effusion with dilated hepatic veins and mild enlargement of the right liver lobe with hyperechoic mesentery (Figure 1). There was decreased blood flow to the right liver lobe using color flow Doppler evaluation. A right liver lobe torsion (LLT)

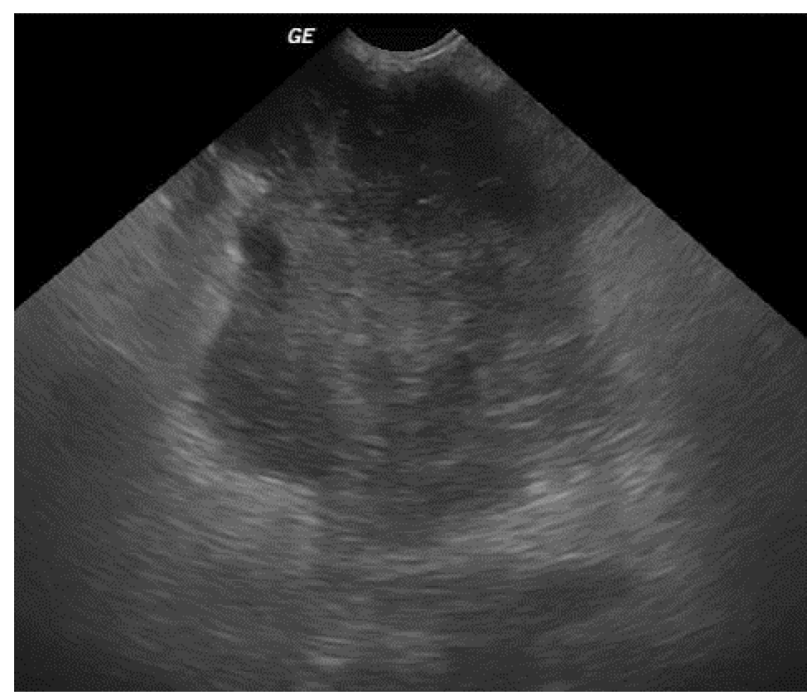

Figure I Transverse ultrasonographic view of the right liver of the dog, showing an extremely heterogeneous and irregularly marginated liver.

Note: Doppler examination of this area revealed decreased blood flow. Abbreviation: GE, general electric.

was suspected. An echocardiogram and computed tomography $(\mathrm{CT})$ were performed prior to surgery to rule out other causes for the pleural effusion.

The echocardiogram revealed mild dilation of the left ventricle with mild mitral and tricuspid regurgitation and a fractional shortening of $32 \%$. There were no significant underlying cardiac abnormalities nor a structural heart disease to account for the pleural effusion or arrhythmia. There were no thoracic or cardiac masses identified or evidence of pericardial effusion. No treatment was warranted based on echocardiogram, and the APCs and VPCs were likely from underlying systemic disease.

The bicavitary CT revealed moderate bilateral pleural effusion and a single pulmonary bullae measuring $0.7 \mathrm{~cm}$ within the dorsolateral aspect of the left caudal lung lobe. The peritoneal effusion had resolved and there was a nonenhancing avascular soft-tissue mass $(11.6 \times 9.8 \times 5.7 \mathrm{~cm})$ with some ring enhancement adjacent to the right limb of the pancreas and caudal to the gallbladder (Figure 2). No cause for pleural effusion was identified.

An exploratory laparotomy was performed. The patient was premedicated with hydromorphone (Hospira Inc., Lake Forest, IL, USA) $0.1 \mathrm{mg} / \mathrm{kg}$ IV and diazepam (Hospira Inc.) $0.25 \mathrm{mg} / \mathrm{kg} \mathrm{IV}$, and induced with etomidate (Amidate; Hospira Inc.) $1 \mathrm{mg} / \mathrm{kg}$ IV. Anesthesia was maintained with isoflurane (Isoflo; Abbot Laboratories) in 100\% oxygen using positive pressure ventilation, and LRS was administered at $10 \mathrm{~mL} / \mathrm{kg} / \mathrm{hr}$ perioperatively.

A standard celiotomy was performed; the caudate liver lobe was noted to be dark purple/black in color and swollen 


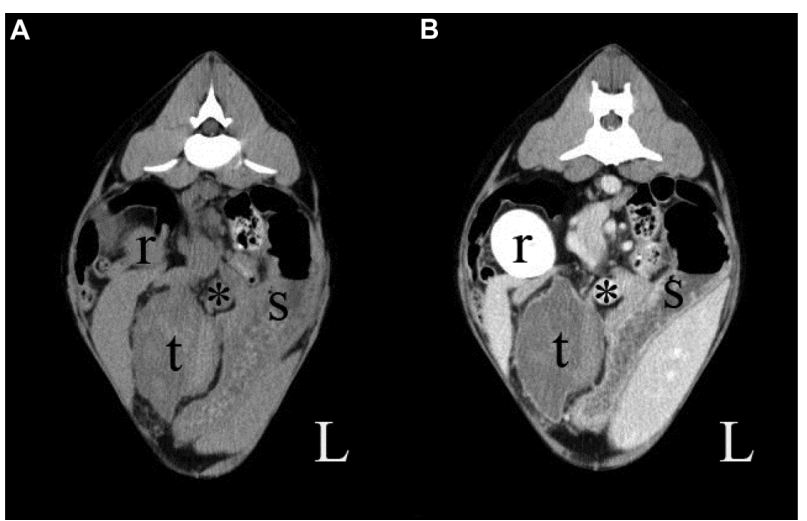

Figure 2 Pre-contrast (A) and post-contrast (B) computed tomography images of the cranial abdomen in soft tissue windows.

Notes: The right kidney (r), stomach (s), and portal vein $(*)$ are present at this level. The torsed liver lobe $(\mathrm{t})$ is irregularly marginated and centrally heterogeneous with poor contrast medium administration; however, it enhances along its peripheral margin with contrast medium. $\mathrm{L}$ indicates the left side of the patient.

with some omental adhesions, and torsed 180 degrees. A liver lobectomy was performed using a thoracoabdominal 55 stapler (Thoracoabdominal ${ }^{\mathrm{TM}} 55$ stapler; Covidien, Mansfield, MA, USA) with $3.5 \mathrm{~mm}$ staples. Medium sized hemoclips and gel foam were used to achieve hemostasis. No other abnormalities were noted within the abdomen. The abdomen was closed with 0 polydioxanone (PDS) for linea alba, 2-0 PDS for subcutaneous layering, and surgical staples for skin closure. Bilateral chest tubes (Argyle trocar catheter $28 \mathrm{fr} \times 16^{\prime \prime}$; Covidien) were placed intraoperatively. Postoperative care included LRS $5 \mathrm{~mL} / \mathrm{kg} / \mathrm{hr}$, hydroxyethyl starch (HESpan; B. Braun Medical Inc., Bethlehem, PA, USA) $1 \mathrm{~mL} / \mathrm{kg} / \mathrm{hr}$, hydromorphone $0.1 \mathrm{mg} / \mathrm{kg}$ IV every 6 hours, metoclopramide (Reglan; Hospira Inc.) $2 \mathrm{mg} / \mathrm{kg} /$ day at a continuous rate infusion, doxycycline (APP Pharmaceuticals LLC, Schaumburg, IL, USA) $5 \mathrm{mg} / \mathrm{kg}$ IV q12h, and enrofloxacin $5 \mathrm{mg} / \mathrm{kg}$ IV q24h.

A recheck $\mathrm{CBC}$ and chemistry on day 1 postoperatively revealed worsening leukocytosis, with a white blood cell count of $27.7 \times 10^{9}$ cells/L (reference interval $5.7 \times 10^{9}$ $16 \times 10^{9}$ cells $\left./ \mathrm{L}\right)$, and neutrophilia with $25.8 \times 10^{9}$ cells $/ \mathrm{L}$ (reference interval $3.0 \times 10^{9}-11.5 \times 10^{9}$ cells/L). The chemistry revealed a worsening hypoalbuminemia of $7 \mathrm{~g} / \mathrm{L}$ (reference interval 22-39 g/L) and total protein of $37 \mathrm{~g} / \mathrm{L}$ (reference interval 52-82 g/L), and improving ALT of $185 \mathrm{U} / \mathrm{L}$ (reference interval 10-100 U/L). Hydroxyethyl starch was increased to $1.5 \mathrm{~mL} / \mathrm{kg} / \mathrm{hr}$ given the hypoalbuminemia.

That evening, the patient had two episodes of sustained ventricular tachycardia (VT) (heart rate of 200-230 beats/min), which responded to lidocaine hydrochloride (Hospira Inc.) $2 \mathrm{mg} / \mathrm{kg}$ IV bolus, and sotalol hydrochloride (Sandoz Inc.,
Princeton, NJ, USA) $1.5 \mathrm{mg} / \mathrm{kg}$ PO q12h was added. The patient continued to have VPCs, but there were no recurrence of the VT during the remainder of the patient's stay.

On day 2 postoperatively, the patient improved and transitioned from hydromorphone to buprenorphine (Reckitt Benckiser Pharmaceutical Inc., Richmond, VA, USA) $0.01 \mathrm{mg} / \mathrm{kg}$. The chest tube was mildly productive immediately postoperatively $(3.5 \mathrm{~mL} / \mathrm{kg} / \mathrm{hr})$. Fluid production gradually decreased to $1.0 \mathrm{~mL} / \mathrm{kg} / \mathrm{hr}$ and chest tubes were pulled later that day.

The following day, mirtazapine (Apotex Inc., Toronto, Canada) $0.6 \mathrm{mg} / \mathrm{kg}$ PO q24h was added, and the patient began eating and was subsequently discharged on sotalol hydrochloride $2 \mathrm{mg} / \mathrm{kg}$ PO q12h, tramadol hydrochloride (Amneal Pharmaceuticals, Hauppage, NY, USA) $2 \mathrm{mg} / \mathrm{kg}$ PO every 8 hours, doxycycline $5 \mathrm{mg} / \mathrm{kg}$ PO $\mathrm{q} 12 \mathrm{~h}$, and mirtazapine $0.6 \mathrm{mg} / \mathrm{kg}$ PO q24h. Given the history of chronic inflammation within the pleural cavity, prednisone (HIKMA Pharmaceuticals, Amman, Jordan) was initiated at $1 \mathrm{mg} / \mathrm{kg}$ q $24 \mathrm{~h}$ PO at a tapering dose for 14 days.

No growth resulted from the aerobic and anaerobic bacterial cultures of the pleural fluid. Resected liver lobe histopathology revealed congestion and hemorrhage with severe segmental ischemic necrosis with peripheral fibroplasia and neovascularization. The focus of necrosis and rarefaction was encapsulated by dense fibrous connective tissue supporting chronicity. There was no evidence of bacteria, neoplasia, or parasitic or fungal organisms.

At follow-up on day 7 postoperatively, the patient was reported to be doing well. Recheck $\mathrm{CBC}$ and chemistry revealed improving leukocytosis of $21.8 \times 10^{9}$ cells/L (reference interval: $5.7 \times 10^{9}-16.3 \times 10^{9}$ cells/L), normal ALT, and mild elevation of alkaline phosphatase of $288 \mathrm{U} / \mathrm{L}$ (reference interval 10-150 U/L).

On day 15 postoperatively, an echocardiogram revealed myxomatous mitral valve degeneration with mild mitral regurgitation, no evidence of tricuspid disease, and complete resolution of the pleural effusion. Recheck electrocardiogram revealed occasional supraventricular and ventricular arrhythmias, but no sustained arrhythmias. Sotalol was continued with no change in the dose.

The owners and patient were relocating abroad and the plan was to follow-up with a cardiologist in England in 1 month. Therefore, further follow-up was not possible. The last communication with the family was 3 weeks postoperatively and clinically the patient was doing well. 
The patient received a high standard of veterinary care and all diagnostics and treatments performed were consented by the owners.

\section{Discussion}

The case presented here describes a patient who presented in respiratory distress for a large volume of pleural effusion, with a subsequent diagnosis of mild peritoneal effusion and LLT. To the authors' knowledge, the case presented here is the first reported case of LLT with pleural and peritoneal effusion (PPE). Differentials for bicavitary effusion include infection, inflammation, bile peritonitis and pleuritis, pancreatitis, cardiac disease, neoplasia, hypoproteinemia, coagulopathy, nonneoplastic liver disease, and trauma. ${ }^{1}$

Liver lobe torsion is an uncommon condition of unknown etiology that has been reported in the $\mathrm{dog},{ }^{2-17} \mathrm{cat},{ }^{13}$ horse,${ }^{18}$ sow, ${ }^{19}$ rabbit, ${ }^{20}$ otter, ${ }^{21}$ and humans. ${ }^{22}$ Clinical signs are nonspecific and may be acute or chronic. ${ }^{2-17}$ Previously reported clinical signs include vomiting, lethargy, anorexia, collapse, diarrhea, polyuria, polydipsia, abdominal pain, abdominal fluid wave, cardiac arrhythmias, weak femoral pulses, palpable abdominal mass, pale mucous membrane, and hyperpyrexia. This patient's clinical signs and physical exam abnormalities included weakness, anorexia, diarrhea, mild fever, respiratory distress, cardiac arrhythmia, heart murmur, and organomegaly.

Dogs with LLT are generally middle-aged to older and usually medium to large body size. There has been one reported case of a small breed dog (ie, Shih-tzu) ${ }^{5}$ with LLT. Although there have been a limited number of LLT cases reported, review of the cases revealed Golden Retrievers as the most common breed followed by Akitas and German shepherds.

Some of the theories postulated for the cause of LLT are ligament aplasia; ${ }^{17}$ gastric dilatation resulting in laxity of the left triangular or hepatogastric ligament; ${ }^{3,10,11}$ severe atrophy of the liver hilus, ${ }^{12}$ abdominal hernia; ${ }^{10}$ and trauma. ${ }^{9,13}$ In the current patient, a cause for the LLT was not found.

Diagnosing LLT can be challenging as hematology and biochemical results are nonspecific. In review of the current literature, elevated ALT, hyperbilirubinemia, and hypoalbuminemia are common in LLT, as was seen in this case.

Radiographs of LLTs can reveal a normal abdomen, a cranial abdominal mass, abdominal effusion, or presence of a gas-filled mass indicating necrosis. Ultrasonography can be a useful modality in evaluating the liver and diagnosing LLT, but it does not provide a definitive diagnosis. On ultrasound, LLT can appear grossly abnormal or as a mass. ${ }^{2,4-6,8-10,12,13,16}$ The mass can be either hypoechoic or have mixed echogenicity; the mass can also be either homogeneous or heterogeneous. Color flow Doppler has been instrumental in identifying LLT in several cases by detecting decreased or no blood flow to the liver. $2,5,6,8,10,16$ The liver can also appear completely normal on ultrasound and diagnoses are made either perioperatively or at necropsy. ${ }^{13}$ In this patient, there was suspicion for LLT based on ultrasound of the liver lobe, which had decreased blood flow and evidence of inflammation.

CT can be a useful diagnostic tool and was chosen in this case given the bicavitary effusion and absence of other causes of pleural effusion. Given the patient's age, underlying neoplasia was a concern, as the pleural effusion was unexpected. The CT in this case showed a necrotic nonenhancing mass, but did not confirm LLT. Differentials for the mass were an infracted/necrotic abscess or tumor arising from the liver, lymph node, or pancreas. However, taken in conjunction with the ultrasound, there was a stronger suspicion for LLT. These findings on ultrasound and CT were similar to previously reported cases that were diagnosed with LLT. ${ }^{5,6}$

In human patients, LLTs are identified by a singular modality or a combination of several modalities. Ultrasonography in conjunction with $\mathrm{CT}$ or magnetic resonance imaging can be utilized to diagnose LLT. However, just as in veterinary patients, diagnoses at times are made perioperatively due to the inconsistencies with the imaging appearance.

It is important to note that although $\mathrm{CT}$, magnetic resonance imaging, and nuclear scintigraphy could assist in diagnosing LLT, it is a surgical emergency, and imaging and/or surgery should not be delayed. Delaying surgery for LLT can lead to additional complications such as systemic inflammatory response syndrome or disseminated intravascular coagulopathy. In the current patient, the owners elected to wait for the fluid analysis prior to pursuing additional diagnostics and then $\mathrm{CT}$ prior to surgery to exclude other causes of pleural effusion.

Pleural effusion as a sequelae of peritoneal effusion has been reported in cats and dogs with cirrhosis, bile peritonitis, pancreatitis, peritoneal dialysis, neoplasia, cardiac diseases, infection, coagulopathy, and trauma. ${ }^{1}$ Patients with LLT commonly present with peritoneal effusion, but pleural effusion has not been reported. Interestingly, the volume of pleural effusion in this case was significant, causing dyspnea, while the peritoneal effusion was mild.

Classification of the pleural effusion is based on fluid cytology. The fluid may be transudate, modified transudate, or exudative. This patient's pleural effusion was classified as exudative (total protein $>30 \mathrm{~g} / \mathrm{L}$ and total nucleated 
cell count $>7 \times 10^{9}$ cells $/ \mathrm{L}$ ) and contained nondegenerate neutrophils and monocytes. Exudative effusions are generally secondary to inflammation or infection resulting from increased capillary permeability (ie, vasculitis), decreased lymphatic removal, or both. Differentials for exudative effusions include neoplasia, infection, LLT, heartworm disease, pneumonia, esophageal diseases, pulmonary thromboembolism, diaphragmatic hernias, uremia, pancreatitis, and lymphoid granulomatosis. ${ }^{23}$ This patient's pleural fluid was consistent with chronic inflammation by the presence of macrophages. This correlates with the patient presenting after 4 days of clinical signs and the liver lobe histopathology supporting chronicity.

In review of the current literature of patients with LLT and peritoneal effusion, the fluid was reported as either modified transudate or exudative. ${ }^{4,6-9,16} \mathrm{An}$ evaluation of the abdominal fluid was not performed in this patient and it would have been useful to confirm that the fluid in the abdomen was comparable to the fluid in the pleural cavity.

In people, PPE is caused by several of the same factors as in dogs. PPE is seen commonly in hemodialysis patients and patients with cirrhosis, pancreatitis, and benign ovarian tumor (Meigs' syndrome), but liver torsion has not been reported in humans as a cause for PPE.

In the current case report, this patient had mild hypoalbuminemia; culture and biopsies did not reveal any evidence of infection; and there was no evidence of significant cardiac disease on echocardiogram. Additionally, other possible causes of PPE like pancreatitis were not seen at the time of surgery. Given the affected liver lobe was right sided, it would have been interesting to measure this patient's canine pancreatic lipase activity. A canine pancreatic lipase activity immunoreactivity was not performed in this patient and the pancreas was grossly normal at surgery.

A possible cause for PPE is peritoneal fluid extending into the thoracic cavity through the diaphragm after being drained by transdiaphragmatic lymphatics or fluid movement into the pleural space through openings around the esophagus and great vessels. Other theories include porous diaphragmatic syndrome ${ }^{24}$ which has been described in people for various disease processes that have resulted in PPE. These are congenital or acquired blisters or blebs in the tendinous portion of the diaphragm. When there is peritoneal fluid present, the diaphragm is stretched and the blisters/blebs rupture allowing unidirectional fluid movement from the abdominal cavity to the pleural space. Due to the unidirectional fluid accumulation into the thorax, some patients have minimal to no peritoneal effusion and significant pleural effusion.
Porous diaphragmatic syndrome has not been reported in the dog, but this mechanism could have played a role since this patient had mild peritoneal effusion with a large volume of pleural effusion.

The most likely mechanism for the bicavitary effusion in this LLT patient is severe inflammation causing the release of inflammatory cytokines and subsequent vasculitis. This could have initiated local and remote inflammation of the transdiaphragmatic lymphatics resulting in an extension of the abdominal disease into the pleural space leading to fluid accumulation.

This theory is further supported by the exudative nature of the pleural fluid, which was most likely the result of permeability of the lymphatic and vascular system rather than increased hydrostatic pressure, which would have resulted in transudative fluid. Furthermore, hypoalbuminemia likely compounded the influx of pleural fluid by decreasing oncotic pressure.

Cardiac arrhythmias have not been reported in human cases of LLT, but have been reported in dogs. ${ }^{2,4,8,10,11}$ VPCs, accelerated idioventricular rhythm (AIVR), and VT can be seen with patients with primary cardiac disease or noncardiac disease. Ventricular arrhythmias are common in hospitalized patients and a frequent occurrence in gastric dilatation-volvulus (GDV) patients. Histological review of cardiac tissue from GDV patients revealed microscopic areas of ischemia most likely secondary to hypovolemia. ${ }^{25}$ It was noted that myocardial ischemia may be a contributing factor, but other factors such as anesthesia and sympathetic tone may also play a role in dysrhythmia. In other patients with abdominal and systemic disease, it is unclear as to the cause of the arrhythmia, but the pathogenesis maybe similar to that of GDV patients.

In one dog, left lateral LLT was suspected to be the cause of both atrial and ventricular escape beats and AIVR. ${ }^{8}$ It was hypothesized that the LLT caused marked increase in afferent vagal stimulation resulting in an arrhythmia. Following surgical removal of the torsed liver lobe, there was spontaneous return to sinus rhythm.

The cardiac arrhythmias in the current patient were APCs, VPCs, AIVR, and VT. Altered vagal stimulation secondary to abdominal disease can explain the arrhythmias, as well as systemic inflammation, and possible ischemia-reperfusion injury. However, unlike the aforementioned patient, evaluation of this patient at 2 weeks revealed continuation of singlet APCs and VPCs despite the removal of the torsed caudate liver lobe. Based on the initial and recheck echocardiograms that were performed, no cardiac cause for the arrhythmia could be seen. As he was lost to follow-up, it is unclear if more time would have allowed resolution of these arrhythmias. 


\section{Conclusion}

This case represents a unique cause of significant pleural effusion with mild peritoneal effusion secondary to LLT. Cases with LLT that are chronic may present with respiratory distress and significant pleural fluid accumulation. Biochemical indicators for LLT are nonspecific, and advanced imaging like ultrasound and CT are usually required for diagnosis preoperatively. Based on current literature, the prognosis for dogs with LLT and subsequent liver lobectomy is excellent. Although LLT occurrences are rare, when assessing patients with pleural effusion, LLT should be considered as a differential diagnosis.

\section{Disclosure}

The authors report no conflicts of interest in this work.

\section{References}

1. Steyn PF, Wittum TE. Radiographic, epidemiologic, and clinical aspects of simultaneous pleural and peritoneal effusions in dogs and cats: 48 cases (1982-1991). J Am Vet Med Assoc. 1993;202(2):307-312.

2. Bhandal J, Kuzma A, Starrak G. Spontaneous left medial liver lobe torsion and left lateral lobe infarction in a rottweiler. Can Vet J. 2008;49(10): 1002-1004.

3. Bronson EM, Coolman BR. A lethargic dog with anorexia and a history of gastric distension. Vet Med. 2002;97:895-898.

4. Downs MO, Miller MA, Cross AR, Selcer BA, Abdy MJ, Watson E. Liver lobe torsion and liver abscess in a dog. J Am Vet Med Assoc. 1998; 212(5):678-680.

5. Lee KJ, Yamada K, Hirokawa H, et al. Liver lobe torsion in a Shih-tzu dog. J Small Anim Pract. 2009;50(3):157.

6. Massari F, Verganti S, Secchiero B, Orifici F, Bonfanti U, Romanelli G. Torsion of quadrate and right middle liver lobes and gallbladder in a German Shepherd dog. Aust Vet J. 2012;90(1-2):44-47.

7. McConkey S, Briggs C, Solano M, Illanes O. Liver torsion and associated bacterial peritonitis in a dog. Can Vet J. 1997;38(7):438-439.
8. Sargent JM, Dennis S, Fuentes VL. ECG of the month. Liver lobe torsion. J Am Vet Med Assoc. 2013;242(6):748-750.

9. Scheck MG. Liver lobe torsion in a dog. Can Vet J. 2007;48(4): 423-425.

10. Schwartz SG, Mitchell SL, Keating JH, Chan DL. Liver lobe torsion in dogs: 13 cases (1995-2004). J Am Vet Med Assoc. 2006;228(2): 242-247.

11. Singh M, Foster D, Marchevsky A, Lamb W. Hepatic lobe torsion and gastric-dilation volvulus in a dog. Aust Vet Pract. 2002;32(4):174-178.

12. Sonnenfield JM, Armbrust LJ, Radlinsky MA, Chun R, Hoskinson JJ, Kennedy GA. Radiographic and ultrasonographic findings of liver lobe torsion in a dog. Vet Radiol Ultrasound. 2001;42(4):344-346.

13. Swann HM, Brown DC. Hepatic lobe torsion in 3 dogs and a cat. Vet Surg. 2001;30(5):482-486.

14. Tomlinson J, Black A. Liver lobe torsion in a dog. JAm Vet Med Assoc. 1983;183(2):225-226.

15. Tubby KG. Concurrent gall bladder, liver lobe torsion, and bile peritonitis in a German shepherd dog 2 months after gastric dilatation/volvulus gastropexy and splenectomy. Can Vet J. 2013;54(8):784-786.

16. von Pfeil DJ, Jutkowitz LA, Hauptman J. Left lateral and left middle liver lobe torsion in a Saint Bernard puppy. J Am Anim Hosp Assoc. 2006;42(5):381-385

17. Woolfe DT, English B. Torsion of the left lateral and papillary lobes of the liver in a pup; a case report. $J$ Am Vet Med Assoc. 1959;134(10):458.

18. Turner TA, Brown CA, Wilson JH, et al. Hepatic lobe torsion as a cause of colic in a horse. Vet Surg. 1993;22(4):301-304.

19. Hamir AN. Torsion of the liver in a sow. Vet Rec. 1980;106(16): 362-363.

20. Weisbroth SH. Torsion of the caudate lobe of the liver in the domestic rabbit (Oryctolagus). Vet Pathol. 1975;12(1):13-15.

21. Warns-Petit ES. Liver lobe torsion in an oriental small-clawed otter (Aonyx cinerea). Vet Rec. 2001;148(7):212-213.

22. Elmasalme F, Aljudaibi A, Matbouly S, Hejazi N, Zuberi MS. Torsion of an accessory lobe of the liver in an infant. J Pediatr Surg. 1995;30(9):1348-1350.

23. Ettinger SJ. Pleural effusion. In: Textbook of Veterinary Internal Medicine. 7th ed. Philadelphia: WB Saunders Co; 2010:266-269.

24. Kirschner PA. Porous diaphragm syndromes. Chest Surg Clin N Am. 1998;8(2):449-472.

25. Muir WW, Weisbrode SE. Myocardial ischemia in dogs with gastric dilatation-volvulus. J Am Vet Med Assoc. 1982;181(4):363-366.
Veterinary Medicine: Research and Reports

\section{Publish your work in this journal}

Veterinary Medicine: Research and Reports is an international, peer-reviewed, open access journal publishing original research, case reports, editorials, reviews and commentaries on all areas of veterinary medicine. The manuscript management system is completely online and includes a very quick and fair peer-review system.

\section{Dovepress}

Visit http://www.dovepress.com/testimonials.php to read real quotes from published authors. 